\title{
Synthesis crystal structure, photoluminescence and photocatalytic property of a new three dimensional Zinc(II) tetrazole framework
}

\author{
ARTI CHOUHAN ${ }^{\mathrm{a}}$, ASHUTOSH PANDEY ${ }^{\mathrm{a}, *}$ and PETER MAYER ${ }^{\mathrm{b}}$ \\ ${ }^{a}$ Department of Chemistry, Motilal Nehru National Institute of Technology, Allahabad 211004, India \\ ${ }^{b}$ Department of Chemistry and Biochemistry, University of Munich, Butenandtstr. 5-13, \\ 81377 Munich, Germany \\ e-mail: apandey@mnnit.ac.in
}

MS received 12 May 2015; revised 16 June 2015; accepted 18 June 2015

\begin{abstract}
In this study the preparation and characterization of a zinc MOF which gives blue luminescence and photocatalytic activity have been described The reaction of 2cyanopyridine, $\mathrm{Zn}\left(\mathrm{NO}_{3}\right)_{2} \cdot 4 \mathrm{H}_{2} \mathrm{O}$ and $\mathrm{NaN}_{3}$ yielded a new $3 \mathrm{D} \mathrm{Zn}(\mathrm{II})$ coordination polymer $\left\{(2 \mathrm{PTZ})_{2} \mathrm{Zn}\right\}_{\mathrm{n}}(\mathbf{1})$, where $2 \mathrm{PTZ}=5$ (2pyridyl)tetrazolate via in situ $[2+3]$ cycloaddition reaction of the nitrile and the azide. Compound $\mathbf{1}$ was characterized by elemental analyses, IR spectroscopy, X-ray powder diffraction (XRPD), scanning electron microscopy (SEM) and singlecrystal X-ray crystallography. Crystal structure of the compound belongs to monoclinic space group C2/c with $Z=2$ In 1 the crosslinkage of the PTZ ligands and Zn(II) atoms forms an infinite 1D chain network. Further the one-dimensional chains are assembled into threedimensional network by hydrogen bonding. Thermal photoluminescence and photocatalytic properties of $\mathbf{1}$ were also been investigated.
\end{abstract}

Keywords. Zinc; 5-(pyridyl)tetrazolato (PTZ); Single-Crystal X-Ray Crystallography.

\section{Introduction}

In last few years tetrazole ligands have been widely used to synthesize metal-organic and H-bonded frameworks of various topologies. ${ }^{1-5}$ The versatility of the tetrazole building blocks provides the required template to create a variety of new materials with interesting properties and diverse applications such as gas storage, ${ }^{6,7}$ sensors, ${ }^{8}$ separation process, ${ }^{9}$ drug delivery, ${ }^{10}$ catalysis, ${ }^{11}$ chemical sensing, ${ }^{12}$ and photocatalysis. ${ }^{13}$ Cycloaddition reactions of azides and cyanides for synthesis of tetrazoles are known for almost 110 years. First report was in 1901 wherein 5 -aminotetrazole was made by the reaction of hydrazoic acid $\left(\mathrm{HN}_{3}\right)$ with cyanamide. ${ }^{14}$ Sharpless et al., in the year 2002, introduced the term "click chemistry" as a chemical philosophy that describes chemistry to be tailored for generating compounds by joining small units together in a practical, quick, and reliable way. ${ }^{15-17}$ Tetrazoles exhibit strong networking ability and in most of the reported MOFs, they usually act as mono- or bidentate ligands. ${ }^{18,19}$ In addition, the five- membered heterocyclic ring could also serve as a hydrogen-bond acceptor, thereby permitting the expansion of the polymeric framework through hydrogen bonding interactions. ${ }^{20}$

\footnotetext{
*For correspondence
}

The factors such as solvent system, $\mathrm{pH}$, coordination behaviour of the metal ion, structural features of the polydentate organic ligand, metal/ligand ratio and the possible second organic ligand deeply affect the self- organization of MOFs. ${ }^{21-23}$ Although in literature a number of MOF materials and metal complexes are reported, research related to luminescent MOFs and complexes is limited especially for ligandbased emissions. ${ }^{24-26}$ Most of the luminescent MOFs attribute the property to the presence of lanthanide elements into their frameworks ${ }^{27}$ either as metal centers or as dopant ions. Ligand-based emissions in MOFs are advantageous because both nature and structure of the linkers can be tuned more effectively in the framework. Such possibilities may prove to be important for the practical application of these materials. Mahata et $a .^{28}$ first reported the use of MOFs as photocatalysts to degrade the organic pollutants. Since then, many MOFs have been excited under UV-visible light \{ owing to the ligand-to-metal-charge-transfer (LMCT) states and used as photocatalysts. ${ }^{29}$ However, most of these MOFs are unstable in water which limits their applications. Interestingly, MOFs based on tetrazole ligands have shown to be highly stable in solvents, particularly in water. It has also been established that MOFs with conjugated organic ligands are promising candidates for photocatalytic applications $^{29}$ due to their luminescent properties. 
In the present work, we report on the reaction of zinc nitrate with 2-cyanopyridine and $\mathrm{NaN}_{3}$ in presence of triethylamine under solvothermal condition at $160^{\circ} \mathrm{C}$ which generated a luminescent 3D coordination polymer, $\left[\mathrm{Zn}(2-\mathrm{PTZ})_{2}\right]_{\mathrm{n}}$, which was structurally characterized by single-crystal $X$-ray diffraction. In addition, the thermal stability, photoluminescence and photocatalytic properties of $\mathbf{1}$ have also been investigated.

\section{Experimental Section}

\subsection{Materials and Methods}

$\mathrm{Zn}\left(\mathrm{NO}_{3}\right)_{2} \cdot 6 \mathrm{H}_{2} \mathrm{O}$ was procured from Merck India. 2Cyanopyridine and sodium azide were procured from Sigma-Aldrich. All the starting reagents were used without further purification. Thermogravimetric analysis data were collected using a Hi-Res TGA 2950 Thermogravimetric analyzer from TA Instruments. C, $\mathrm{H}$ and $\mathrm{N}$ analyses were performed on a Perkin-Elmer 2400 CHN Elemental Analyzer. Fourier transform infrared (FTIR) spectroscopy was done on a Jasco FT-IR-5300 spectrophotometer (as $\mathrm{KBr}$ pellet) within 4000-400 $\mathrm{cm}^{-1}$ region. The photoluminescence spectrum was recorded on an ocean optics system within 385-750 nm range.

\subsection{Synthesis of 1}

A mixture of $\mathrm{Zn}\left(\mathrm{NO}_{3}\right)_{2} \cdot 6 \mathrm{H}_{2} \mathrm{O}$ (298 mg, 1mmol), 2cyanopyridine (208 mg, $2 \mathrm{mmol}$ ), sodium azide (195 $\mathrm{mg}, 3 \mathrm{mmol})$, triethylamine $(202 \mathrm{mg}, 2 \mathrm{mmol})$ and $15 \mathrm{~mL}$ of DMF was placed in a $20 \mathrm{~mL}$ Teflon-lined stainless-steel reactor and heated at $160^{\circ} \mathrm{C}$ for $24 \mathrm{~h}$, under autogenous pressure and then brought to room temperature at a rate of $5^{\circ} \mathrm{C} / \mathrm{h}$. Colourless crystals suitable for X-ray analysis were obtained in a good yield (71\% based on metal salt). Microanalysis $-\mathrm{C}_{12} \mathrm{H}_{8} \mathrm{~N}_{10} \mathrm{Zn}$ (357.65): calcd. (\%) C 40.37, H 2.04, N 39.21, Found (\%) C 40.45, H 2.43, N 40.01. Selected peaks in IR spectrum $\left(\mathrm{KBr}, \mathrm{cm}^{-1}\right)$ : $1638(\mathrm{w}), 1612(\mathrm{~s}), 1571(\mathrm{~m})$, 1469 (m), 1444 (s), 1400 (m), 1138 (m), 757 (m), 732 (m), $513(\mathrm{w})$.

\subsection{Structure Determination and Refinement}

A single crystal suitable for X-ray crystallography was mounted on a nylon loop. Single-crystal X-ray data of 1 were collected at 173(2) K by using a Bruker TXS diffractometer with $\mathrm{Mo} \mathrm{K} \alpha$ radiation $(k=0.71073$ $\AA$ ) The structure was solved by direct methods with SIR97 $7^{30}$ and refined through full-matrix least squares techniques on $\mathrm{F}^{2}$ by the SHELX-97 program. ${ }^{31}$ All

Table 1. Crystallographic data of compounds $\mathbf{1}$.

net formula

$M_{\mathrm{r}} / \mathrm{g} \mathrm{mol}^{-1}$

crystal size/mm

$T / \mathrm{K}$

radiation

diffractometer

crystal system

space group

$a / b / c / \AA$

$\alpha / \beta / \gamma^{\circ}$

$V / \AA^{3}$

$Z$

calc. density/g $\mathrm{cm}^{-3}$

$\mu / \mathrm{mm}^{-1}$

absorption correction

transmission factor range

refls. measured

$R_{\text {int }}$

mean $\sigma(I) / I$

$\theta$ range

observed refls.

$x, y$ (weighting scheme)

hydrogen refinement

refls in refinement

parameters

$R\left(F_{\text {obs }}\right), R_{\mathrm{W}}\left(F^{2}\right)$

$S$

Symmetry code for figure: $\mathrm{i}=1-x, y, 1 / 2-z ; \mathrm{ii}=x,-y$, $z-1 / 2 ;$ iii $=1-x,-y, 1-z$.

${ }^{\mathrm{a}} R 1(F)=\Sigma \| F_{\mathrm{o}}|-| F_{\mathrm{c}}|/| \Sigma\left|F_{\mathrm{o}}\right| ;{ }^{\mathrm{b}} w R 2\left(F^{2}\right)=\left[\Sigma w\left(F_{\mathrm{o}}^{2}-\right.\right.$ $\left.\left.F_{\mathrm{c}}^{2}\right)^{2} / \Sigma w\left(F_{\mathrm{o}}^{2}\right)^{2}\right]^{1 / 2} ;{ }^{\mathrm{c}} S=\mathrm{GoF}=\left[\Sigma w\left(F_{\mathrm{o}}^{2}-F_{\mathrm{c}}^{2}\right)^{2} /\left(n_{\mathrm{obs}}-\right.\right.$ $\left.\left.n_{\text {param }}\right)\right]^{1 / 2} ; \quad w=\left[\sigma^{2}\left(F_{\mathrm{o}}^{2}\right)+(a P)^{2}+b P\right]^{-1}$, where $P=$ $\left(\operatorname{Max}\left(F_{\mathrm{o}}^{2}, 0\right)+2 F_{\mathrm{c}}^{2}\right) / 3$.

non-hydrogen atoms were refined anisotropically, while the hydrogen atoms were located geometrically and refined isotropically. Pertinent crystal data and structural refinement for $\mathbf{1}$ are summarized in table 1 .

\section{Results and Discussion}

The IR spectrum of $\mathbf{1}$ exhibits several characteristic strong and medium bands There are no absorptions around $\sim 2300 \mathrm{~cm}^{-1}$ and $\sim 2100 \mathrm{~cm}^{-1}$ indicating the absence of cyano and azide groups while the peaks in the range 1571-1447 $\mathrm{cm}^{-1}$ indicate that the [2+3] cycloaddition reaction between cyano group and azide anion has taken place.

\subsection{Crystal Structure Description of 1}

The single-crystal X-ray structural analysis reveals that $\mathbf{1}$ is a three-dimensional framework. The asymmetric 
Table 2. Selected bond angles $\left({ }^{\circ}\right)$ and Bond Distances $(\AA)$ for compound $\mathbf{1}$.

\begin{tabular}{lllcccc}
\hline Atom1 & Atom2 & Atom3 & Angle & Atom1 & Atom2 & Length \\
\hline N2 & Zn1 & -N2i & $90.59(10)$ & Zn1 & N2 & $2.1249(19)$ \\
N1ii & $-Z n 1$ & $-N 2 i$ & $95.74(7)$ & Zn1 & N1ii & $2.1119(17)$ \\
N2 & $-Z n 1$ & -N5iii & $172.03(6)$ & Zn1 & N5ii & $2.2259(19)$ \\
N2 & - Zn1 & -N5ii & $90.28(7)$ & N5 & C2 & $1.348(3)$ \\
N1ii & $-Z n 1$ & -N5ii & $76.32(7)$ & N5 & C6 & $1.335(3)$ \\
\hline
\end{tabular}

unit contains one $\mathrm{Zn}$ (II) cation on a special position and one tetrazole ligand. The $\mathrm{Zn}$ (II) cation is located on a twofold axis of the space group $\mathrm{C} 2 / \mathrm{c}$ leading to a $\mathrm{C}_{2}$-symmetric octahedral coordination sphere consisting of four tetrazole nitrogen atoms (two N1 and two N2) and two pyridyl nitrogen atoms (two N5) provided by four PTZ ligands. The Zn-N1 [2.1119(17) A] and $\mathrm{Zn}-\mathrm{N} 2$ [2.1249(19) $\mathrm{A}]$ distances all fall into the normal bond length ranges i.e., 2.113 to $2.154 \AA$, corresponding to the typical values in $\mathrm{Zn}$ (II)-nitrogen compounds, ${ }^{23,32}$ but the $\mathrm{Zn}-\mathrm{N} 5$ distance $[2.2259(19) \AA]$ is slightly longer. The bond angles $\mathrm{N}-\mathrm{Zn} 1-\mathrm{N}$ are in the range from $76.32(7)^{\circ}$ to $97.11(11)^{\circ}$ for cis-standing nitrogen atoms, while they are $172.03(6)^{\circ}$ and $170.87(10)^{\circ}$ for trans-standing nitrogen atoms. Selected bond lengths and angles are given in table 2.

The rings of the PTZ ligand are not coplanar and form a dihedral angle of $12.15(12)^{\circ}$ (figure 1). PTZ acts as a tridentate ligand and provides two nitrogen donors to one zinc centre, both from the pyridyl as well as the tetrazole group, while the one nitrogen of the tetrazole group coordinates to an adjacent zinc atom. Thus, each PTZ coordinates to two different zinc centres forming a $\mathrm{M}_{2} \mathrm{~L}_{4}$ type coordination polymer along [001] $]^{33}$ (figures $2 \mathrm{a}$ and $2 \mathrm{~b}$ ).

In the packing of 1 , weak contacts of the type C3$\mathrm{H} 3 \cdots \mathrm{N} 4$ with a H-N distance of $2.475 \AA$ and an C$\mathrm{H}-\mathrm{N}$ angle of $172^{\circ}$ exist between pyridyl $\mathrm{C}-\mathrm{H}$ groups

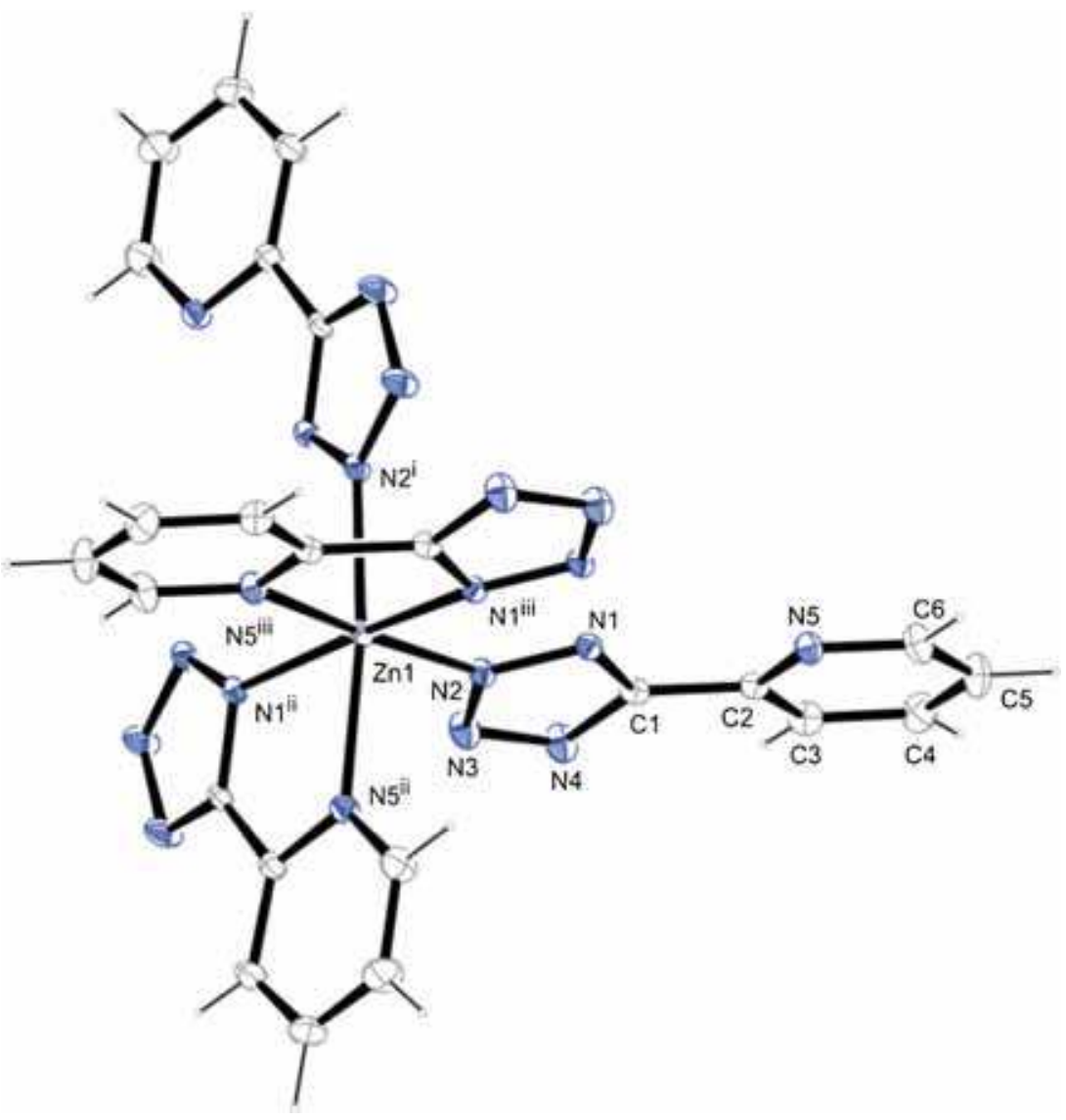

Figure 1. Coordination environment of $\mathrm{Zn}(\mathrm{II})$ ion in $\mathbf{1}$. 

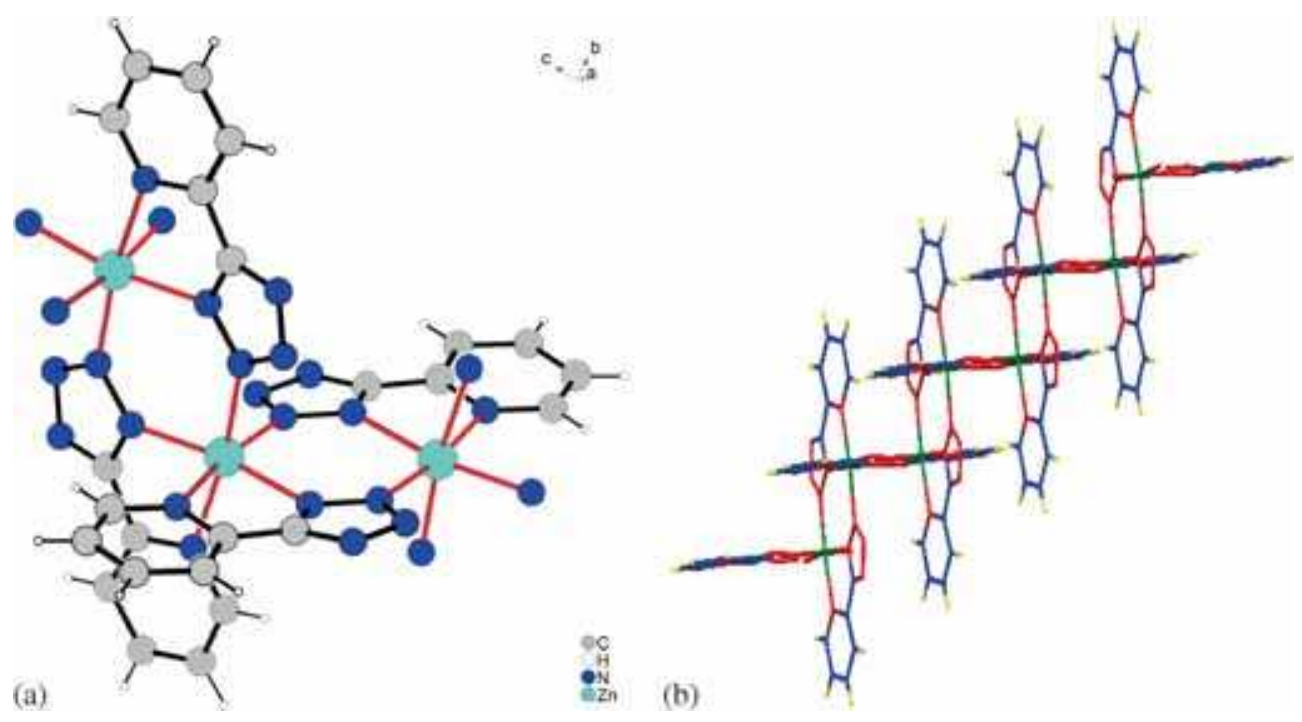

Figure 2. (a) $\mathrm{M}_{2} \mathrm{~L}_{4}$ type coordination polymer of $\mathbf{1}$, and (b) $1 \mathrm{D}$ chain to form a staircase like structure along [001] direction.

and tetrazolyl nitrogen atoms. By two of these contacts ten-membered rings consisting of two donor and two acceptor atoms are formed which can be described with a $R_{2}^{2}(10)$ descriptor in terms of graph-set analysis ${ }^{34,35}$ (figures $3 a$ and $3 b$ ).

It is still a challenge for chemists to design and construct MOFs with the expected structures and properties, because the molecular structural architectures of MOFs are influenced by various factors, such as the reaction temperature and $\mathrm{pH}$ value. A trinuclear cadmium complex, $\mathrm{Cd}_{3}(\mathrm{OH})_{2} \mathrm{Cl}_{2}(4-\mathrm{PTZ})_{2}$, has been reported by Xue et al. which was obtained by the reaction of cadmium chloride with 4-cyanopyridine in the presence of $\mathrm{NaN}_{3}$ and water $(p \mathrm{H} \sim 6.5)$ under hydrothermal condition at $110^{\circ} \mathrm{C}$ (via in situ generation of the 4-PTZ ligand). ${ }^{36}$ On the other hand, a mononuclear cadmium complex, $\mathrm{Cd}(4-\mathrm{PTZ})_{2}\left(\mathrm{H}_{2} \mathrm{O}\right)$, was carried out by the same group wherein reaction of $\mathrm{CdCl}_{2}$ in $\mathrm{H}_{2} \mathrm{O}$ was carried out with pre-synthesized 4-PTZ ( $p \mathrm{H} \sim 4.0$ ) under the same hydrothermal conditions. The authors concluded that $p \mathrm{H}$ is responsible for the difference. The structure of previously reported $\mathrm{Zn}(\mathrm{II})$ complex with the same ligand (2-PTZ) i.e., $\left[\mathrm{Zn}(2-\mathrm{PTZ})_{2}\left(\mathrm{H}_{2} \mathrm{O}\right)_{2}\right]_{\mathrm{n}}{ }^{18}$ is quite different from the structure of $\mathbf{1}$. The complex $[\mathrm{Zn}(2-$ $\left.\mathrm{PTZ})_{2}\left(\mathrm{H}_{2} \mathrm{O}\right)_{2}\right]_{\mathrm{n}}$ was synthesized by a $[2+3]$ cycloaddition reaction under hydrothermal heating at $105^{\circ} \mathrm{C}$ with 2-cyanopyridine, $\mathrm{NaN}_{3}$ and $\mathrm{ZnCl}_{2}(p \mathrm{H} \sim 6.5)$. The complex $\left[\mathrm{Zn}(2-\mathrm{PTZ})_{2}\left(\mathrm{H}_{2} \mathrm{O}\right)_{2}\right]_{\mathrm{n}}$ crystallizes as a two-dimensional chain with four equatorial nitrogen atoms from two 2-PTZ ligands and two apical water molecules, which was supported by Sharpless's proposed structures of the intermediate precipitates. ${ }^{15-17}$ The reaction of zinc nitrate, 2-cyanopyridine and
$\mathrm{NaN}_{3}$ in presence of triethylamine under solvothermal condition (present work) at $160^{\circ} \mathrm{C}(p \mathrm{H} \sim 8.5)$ leads to the formation of $\left[\mathrm{Zn}(2-\mathrm{PTZ})_{2}\right]_{\mathrm{n}}$. This result has further substantiated the mechanism proposed by Sharpless's for the formation of tetrazoles. The compositional and structural difference between $[\mathrm{Zn}(2-$ PTZ $\left.)_{2}\left(\mathrm{H}_{2} \mathrm{O}\right)_{2}\right]_{\mathrm{n}}$ and $\mathbf{1}$ is probably due to the different thermal conditions and $p \mathrm{H}$ of their respective reactants mixtures.

\section{$3.2 X R P D, S E M$}

The XRPD pattern of compound $\mathbf{1}$ was investigated at room temperature. The average crystallite size calculated by Scherrer equation using four strongest reflections (corresponding to (110), (020), (221) and (331)) in $\mathbf{1}$ is $\sim 72 \mathrm{~nm}$. As shown in figure 4, the XRPD patterns measured for $\mathbf{1}$ were in good agreement with the XRPD patterns simulated from the respective single-crystal X-ray data of $\mathbf{1}$ using the Mercury 1.4 program, demonstrating the phase purity of the product. The dissimilarities in intensities may be due to the preferred orientations in the crystalline powders.

Figure 5 shows SEM image of the as-synthesized crystals of compound $\mathbf{1}$ which demonstrates that morphologically, the product consists of 3D flowerlike microspheres with diameters ranging from 30 to $40 \mu \mathrm{m}$. Interestingly, these flower-like microspheres are built from densely packed layers of two dimensional microflakes with a thickness of about 700-800 nm. A close observation reveals thatthese densely packed microflakes bloom out from the central part and appear like a flower. 


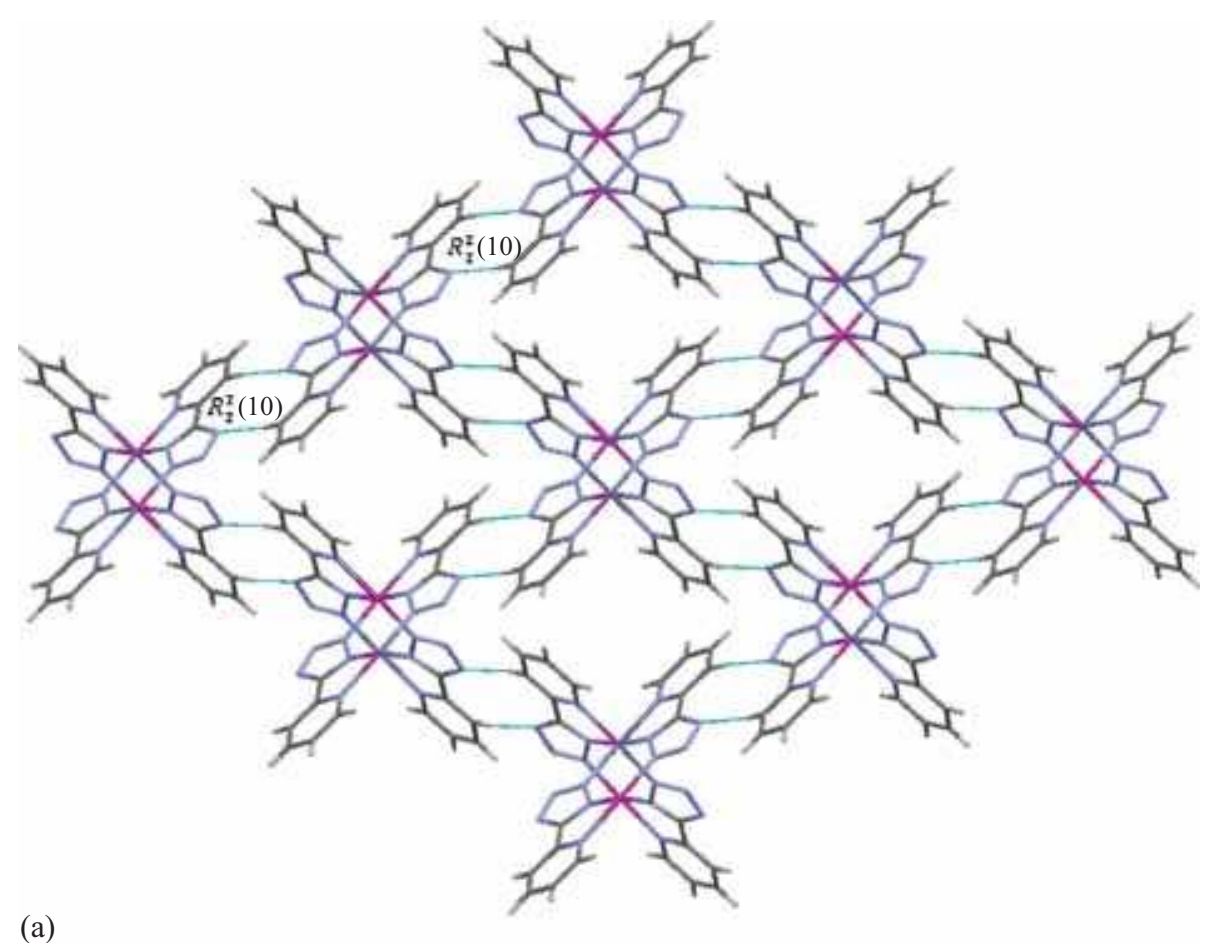

(a)

(b)

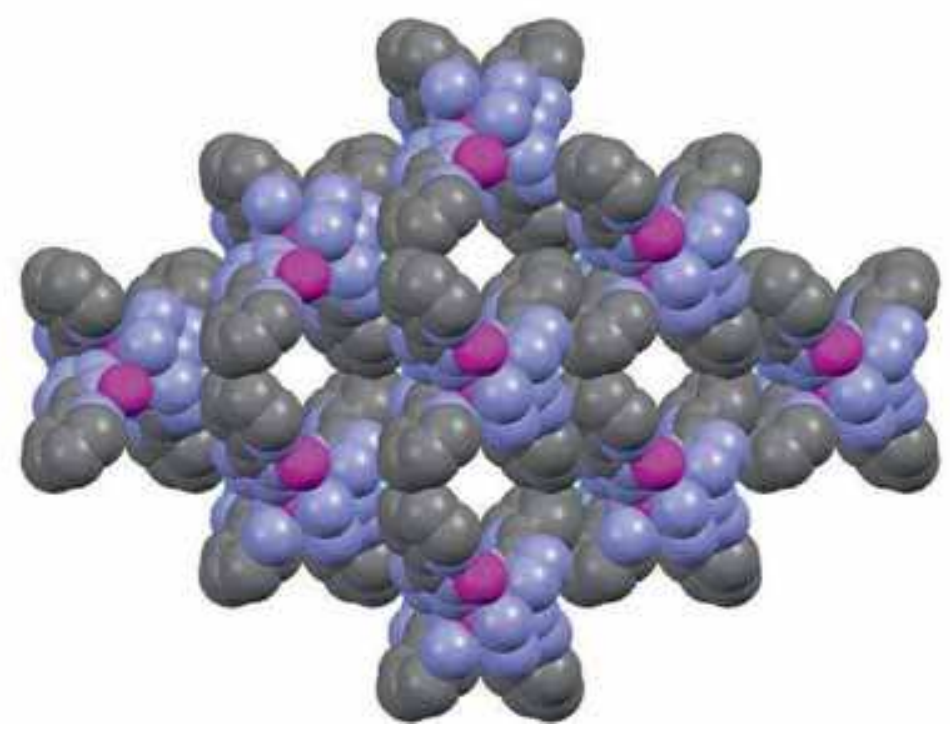

Figure 3. (a) View of the crystal lattice showing H-bonded tapes based on $\mathrm{R}_{2}^{2}(10)$ motifs; (b) space filled diagram.

\subsection{UV-Vis diffuse reflectance spectroscopy and Band-gap calculation}

The diffuse-reflectance UV/Vis spectrum shows the absorption feature (figure 6) of $\mathbf{1}$. The main UV absorption band is around $398 \mathrm{~nm}$, which can be assigned to ligand-to-metal charge transfer (LMCT). To obtain the precise value of band gap from the absorption edge, the point of inflection in the first derivative of the absorption spectrum was used. The value of the band gap obtained from the corresponding transition is 3.2
eV. Band gap was calculated by using the Tauc plot showing in figure 6 (inset).

\subsection{Photocatalytic property}

The photocatalytic activity of $\mathbf{1}$ was evaluated by the degradation of Methylene Blue (MB) in aqueous solution. Experimental details for photocatalysis are available in Supplementary Information. The photodegradation experiment under UV light irradiation was carried out after the dark adsorption equilibrium was achieved. 


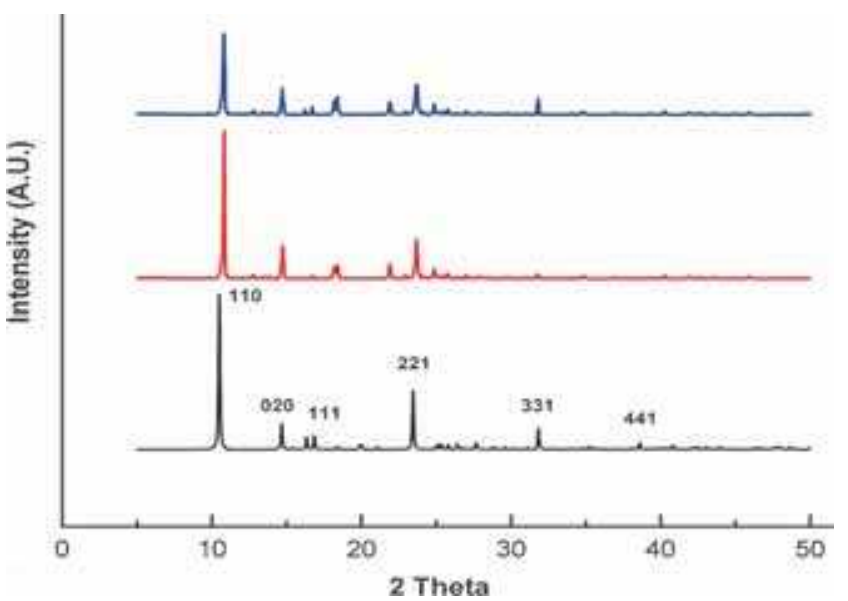

Figure 4. X-ray powder diffraction diagram of the simulated spectra from single crystal data of $\mathbf{1}$ (black), assynthesized 1 (red), and after photocatalytic measurement (blue).

The characteristic absorption of MB at about $650 \mathrm{~nm}$ was selected to monitor the adsorption and photocatalytic degradation process (figure S1 in Supplementary Information). The comparison of decomposition rate of $\mathrm{MB}$ under UV light in the presence and absence of $\mathrm{H}_{2} \mathrm{O}_{2}$ using compound $\mathbf{1}$ as photocatalyst is depicted in figure 7. The control experiments show that no $\mathrm{MB}$ degradation was observed over the photocatalyst $\mathbf{1}$ and $\mathrm{H}_{2} \mathrm{O}_{2}$ without the light irradiation (figure $7 \mathrm{e}$ ). In contrast, $\sim 2 \% \mathrm{MB}$ was self-decolourised after $20 \mathrm{~min}$ of UV irradiation (figure $7 \mathrm{a}$ ), while $\sim 25 \% \mathrm{MB}$ was degraded after $20 \mathrm{~min}$ (figure $7 \mathrm{c}$ ). Thus, it can be concluded that 1 shows the photocatalytic activity for MB degradation, although the photodegradation rate is low.

The photocatalytic activity of $\mathbf{1}$ for MB degradation can be enhanced by the addition of the hydrogen peroxide $\left(\mathrm{H}_{2} \mathrm{O}_{2}\right)$ electron acceptor. ${ }^{29}$ As shown in scheme 1 ,

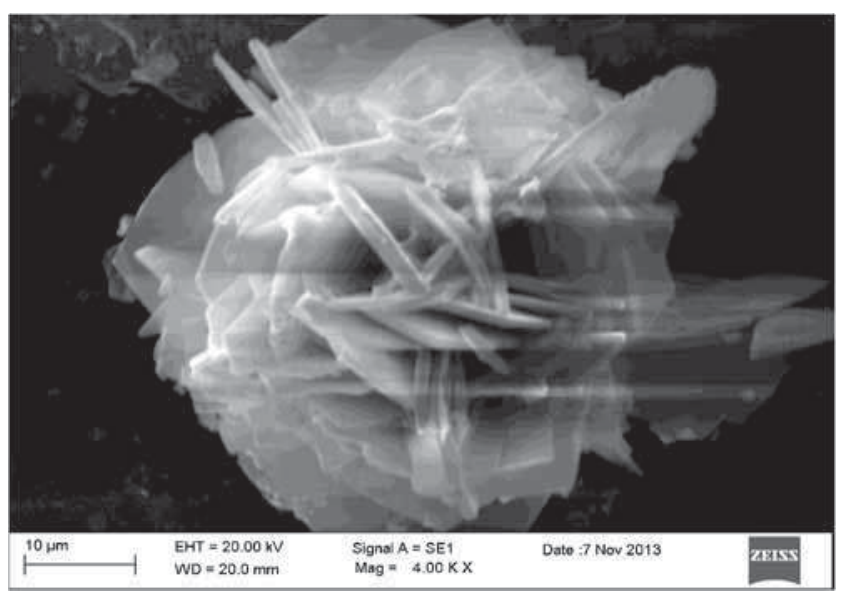

Figure 5. SEM image of compound 1 showing floral arrangement.

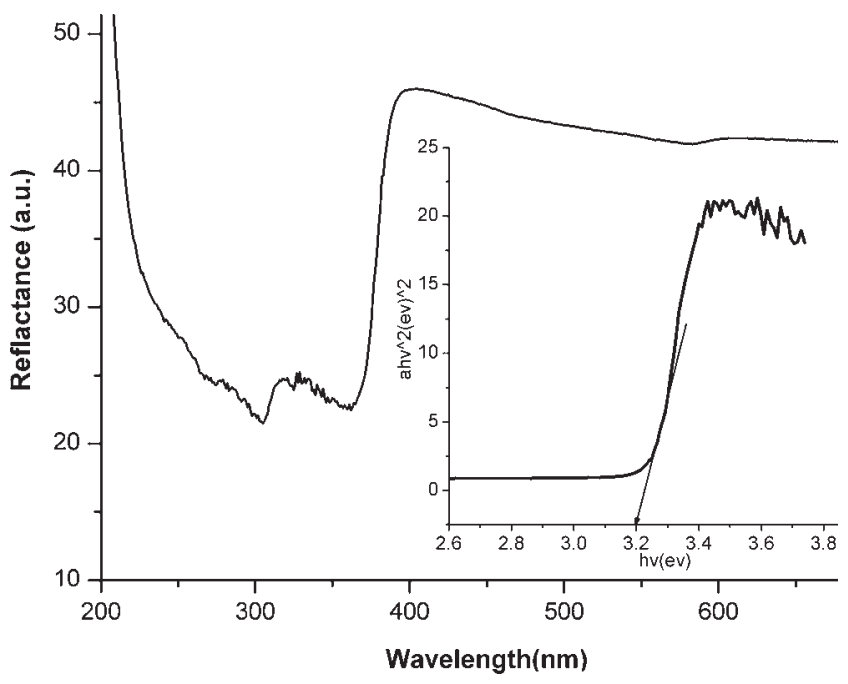

Figure 6. UV-VIS diffuse reflectance spectrum. Inset shows the Tauc plot of sample $\mathbf{1}$.

the photocatalytic reaction starts from the generation of electron-hole pairs. The photoexcited holes either react with organic moieties directly or react with $\mathrm{OH}^{-}$ to generate $\mathrm{OH}$ for further oxidation reaction. The electron-hole recombination reduces the efficiency of photocatalytic reaction. Therefore, the introduction of an external electron acceptor is expected to suppress the electron-hole recombination and enhance the photocatalytic efficiency. An enhanced photodegradation efficiency with the addition of $\mathrm{H}_{2} \mathrm{O}_{2}$ as an electron acceptor can be explained by eq. $\mathrm{H}_{2} \mathrm{O}_{2}+\mathrm{e}^{-} \rightarrow \mathrm{OH}^{-}+\mathrm{OH}$. 20 min UV irradiation induces $70 \% \mathrm{MB}$ degradation (figure $7 \mathrm{~d}$ ), whereas less than $50 \% \mathrm{MB}$ was degraded when only $\mathrm{H}_{2} \mathrm{O}_{2}$ was used in the reaction (figure $7 \mathrm{~b}$ ).

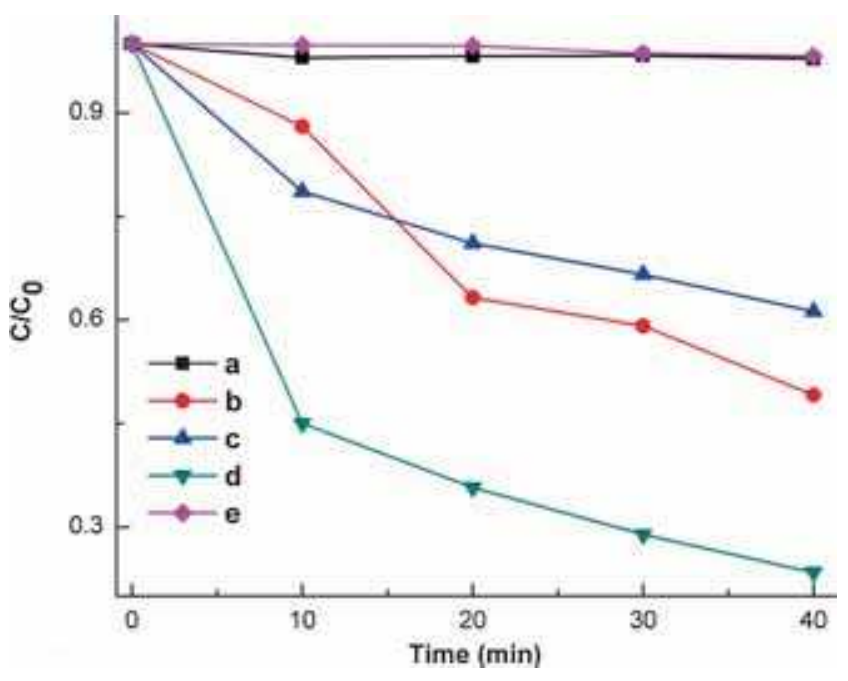

Figure 7. $\mathrm{MB}$ decolorization profiles with UV irradiation on different conditions. (a) MB self-decolorization; (b) with $\mathrm{H}_{2} \mathrm{O}_{2}$ as electron acceptor; (c) using $\mathbf{1}$ as the photocatalyst; (d) with $\mathrm{I}$ as photocatalyst and $\mathrm{H}_{2} \mathrm{O}_{2}$ as electron acceptor; and (e ) with $\mathbf{1}$ and $\mathrm{H}_{2} \mathrm{O}_{2}$ but without UV irradiation. 


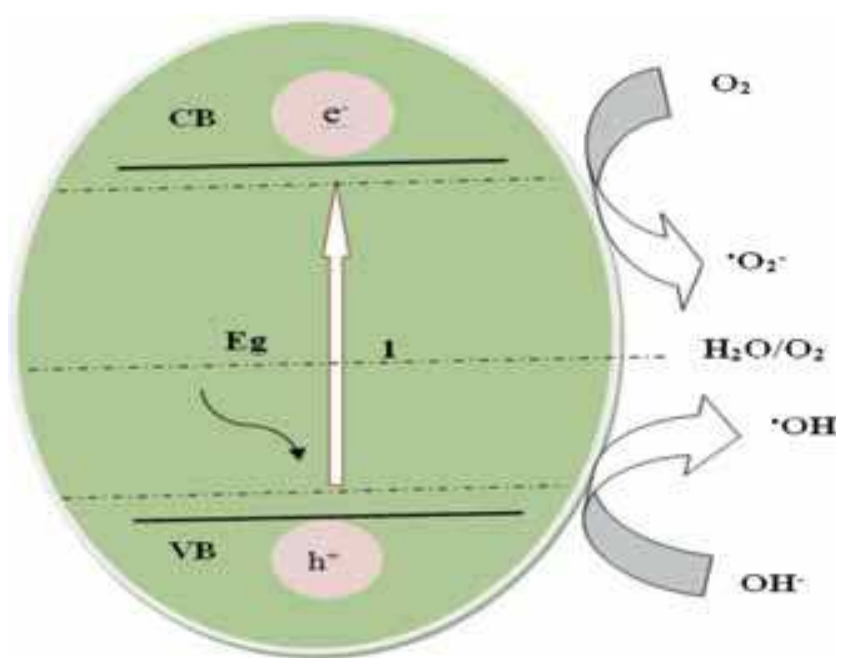

Scheme 1. Schematic representation of photocatalytic mechanism of $\mathbf{1}$ using $\mathrm{H}_{2} \mathrm{O}_{2}$.

In addition, stability of compound $\mathbf{1}$ was monitored using XRPD during course of the photocatalytic reaction (figure 5). After photocatalysis, the XRPD pattern of compound $\mathbf{1}$ is slightly different to that of the original compound implying its stability towards photocatalytic reactions.

\subsection{Thermal Analysis}

Compound $\mathbf{1}$ is air-stable and retains crystalline integrity at ambient temperature. The thermogravimetric analysis curve (figure 8) shows it is stable up to $240^{\circ} \mathrm{C}$ and thereafter the organic groups starts to decompose. The weight loss between $231^{\circ} \mathrm{C}$ to $275^{\circ} \mathrm{C}$ has been attributed to the decomposition of three molecules of $\mathrm{N}_{2}$ (calc. $26.61 \%$, obs.25.57\%). In the temperature range $383^{\circ} \mathrm{C}$ to $457^{\circ} \mathrm{C}$ two cyano-pyridine groups were liberated (calc. $80.54 \%$, obs. $76.92 \%$ ).

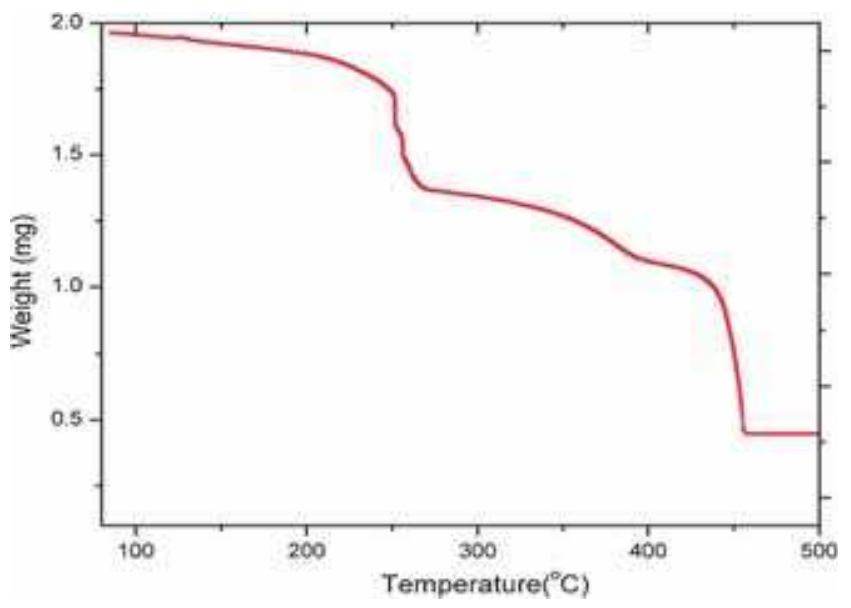

Figure 8. Thermogravimetric analysis (TGA) for $\mathbf{1}$.

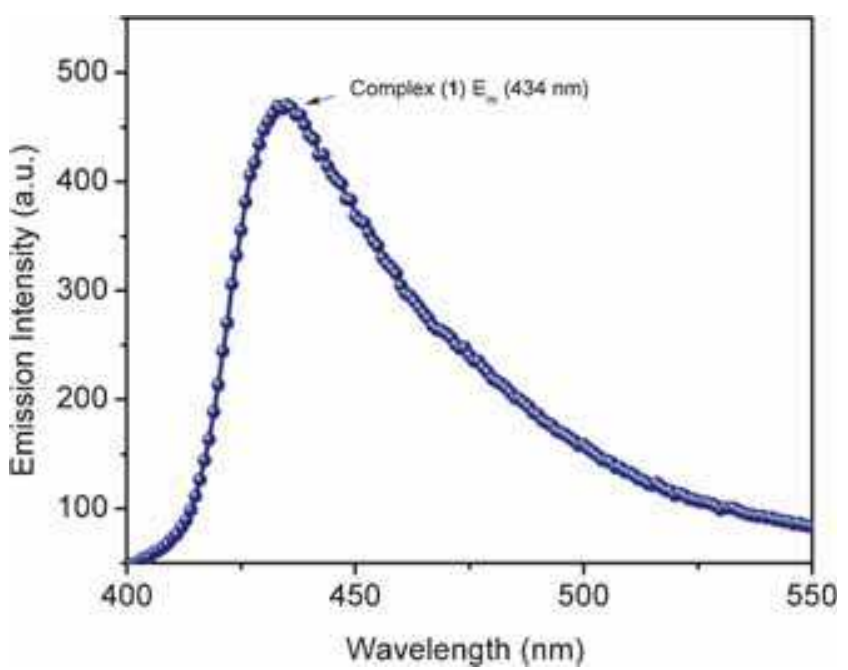

Figure 9. The emission spectrum of $\mathbf{1}$ in solid state at room temperature.

These consecutive decompositions suggest the total destruction of the framework.

\subsection{Photoluminescence}

Subsequently, fluorescence of compound $\mathbf{1}$ has been investigated at room temperature in the solid state. As shown in figure 9, 1 exhibits a strong emission band at $454 \mathrm{~nm}$ upon excitation at $357 \mathrm{~nm}$. The emission of compound 1 may be attributed to the $\pi_{\mathrm{tz}^{-}}$ 5s LMCT transition. ${ }^{25}$ Thus, compound $\mathbf{1}$ may find potential applications as a luminescent material in blue light-emitting devices.

\section{Conclusions}

In conclusion, a new bis[5-(2-pyridyl)tetrazolato]zinc (II) coordination polymer has been synthesized in situ under hydrothermal conditions and characterized by single crystal X-ray structure determination. Compound 1 features a 3D infinite coordination network of dimeric $\left\{(2-\mathrm{PTZ})_{2} \mathrm{Zn}\right\}$ repeat units with octahedral coordination around zinc centers. In addition, it displays modest thermal stability and shows strong solid-state blue fluorescent emission. Lastly, compound $\mathbf{1}$ is a photocatalyst and decolourizes methylene blue under UV irradiation.

\section{Supplementary Information}

Supplementary crystallographic data for $\mathbf{1}$ have been deposited with the CCDC 985644 and can be obtained free of charge from The Cambridge Crystallographic Data Centre via http://www.ccdc.cam.ac. uk/Community/Requestastructure/Pages/DataRequest. 
aspx. (or from the Cambridge Crystallographic Data Centre, 12, Union Road, Cambridge, CB2 1EZ, UK (fax: +44 (1223) 336-033; e-mail: deposit@ccdc.cam.ac.uk). Other supporting information associated with this article i.e., figure $\mathrm{S} 1$ and photocatalytic experimental details are available in the electronic form of supplementary data. Supplementary Information is available at www.ias.ac.in/chemsci.

\section{Acknowledgements}

The authors gratefully acknowledge the CIR facility of MNNIT, Allahabad.

\section{References}

1. Liu W T, Li J Y, Ni Z P, Bao X, Leng J D, Liu J L and Tong M L 2012 Cryst. Growth Des. 121482

2. Cui P, Chen Z, Gao D, Zhao B, Shi W and Cheng P 2010 Cryst. Growth Des. 104370

3. Nasani R, Saha M, Mobin S M and Mukhopadhyay S 2013 Polyhedron 5524

4. Singh S, Gupta D K, Noeth H and Pandey A 2013 J. Chem. Crystallogr. $\mathbf{4 3} 82$

5. Gupta D K, Singh S, Mayer P and Pandey A 2011 Inorg. Chem. Com. 141485

6. Suh M P, Park H J, Prasad T K and Lim D W 2012 Chem. Rev. 112(2) 782

7. He Y B, Zhou W, Yildirim T and Chen B L 2013 Energy Environ Sci. 62735

8. Kreno L E, Leong K, Farha K, Allendorf M, Duyne V and Hupp T 2012 Chem. Rev. 1121105

9. Lin J B, Xue W, Zhang J P and Chen X M 2011 Chem. Commun. 47926

10. McKinlay A C, Morris R E, Horcajada P, Ferey G, Gref R, Couvreur P and Serre C 2010 Angew. Chem. Int. Ed. 496260

11. Wanderley M M, Wang C, Wu C D and Lin W B 2012 J. Am. Chem. Soc. 134(22) 9050

12. Wang G Y, Yang L L, Li Y, Song H, Ruan W J, Chang $\mathrm{Z}$ and $\mathrm{Bu}$ X H 2013 Dalton Trans. 4212865

13. Zhang T and Lin W 2014 Chem. Soc. Rev. 435982

14. Hantzsch A and Vagt A 1901 Ueber das sogenannte Diazoguanidin. Liebig's Ann 314339

15. Demko Z P and Sharpless K B 2001 J. Org. Chem. 66 7945
16. Demko Z P and Sharpless K B 2002 Angew. Chem. 114 2214; 2002 Angew. Chem. Int. Ed. 412110

17. Himo F, Demko Z P, Noodleman L and Sharpless K B 2003 J. Am. Chem. Soc. 1259983

18. Wang L Z, Qu Z R, Zhao H, Wang X S, Xiong R G and Xue Z L 2003 Inorg. Chem. 423969

19. Singh S, Mayer P and Pandey A 2010 Indian J. Chem. 49A 1345

20. Wu J Y, Huang S M, Huang Y C and Lu K L 2012 Cryst. Eng. Comm. 141189

21. Robson R, Abrahams B F, Batten S R, Gable R W, Hoskins B F and Liu J 1992 ACS Symp. Ser. T Bein (Ed.) (ACS: Washington, DC) 499256

22. Haasnoot G J 2000 Coord. Chem. Rev. 200131

23. Su C Y, Cai Y P, Chen C L, Smith M D, Kaim W and Loye H C 2003 J. Am. Chem. Soc. 1258595

24. Bauer A, Timofeeva V, Settersten B, Patterson D, Liu H, Simmons A and Allendorf D 2007 J. Am. Chem. Soc. 1297136

25. Mishra A, Nayak P K and Periasamy N 2004 Tetrahedron Lett. 456265

26. Nayak P K, Agarwal N, Ali F, Patankar M P, Narasimhan K L and Periasamy N J. Chem. Sci. 122 847

27. Fordham S, Wang X, Bosch M and Zhou H 2015 "Lanthanide Metal-Organic Frameworks" In Structure and Bonding P Cheng (Ed.) (Berlin: Heidelberg) 163 p. 1

28. Mahata P, Madras G and Natarajan S 2006 J. Phys. Chem. B 11013759

29. Wang C C, Li J R, Lv X L, Zhang Y Q and Guo G 2014 Energy Environ. Sci. 72831

30. Altomare A, Burla M C, Camalli M, Cascarano G L, Giacovazzo C, Guagliardi A, Moliterni A G G, Polidori G and Spagna R 1999 J. Appl. Cryst. 32115

31. Sheldrick G M 1997 SHELXL-97: Program for refining crystal structure refinement (University of Goettingen: Goettingen)

32. Zhu H B, Yang W N and Hu J 2013 J. Coord. Chem. 16 2775

33. Ma H, Xu Y, Meng Q, Zhang L, Wang R and Sun D 2014 Z. Anorg. Allg. Chem. 6401219

34. Bernstein J, Davis R E, Shimoni L and Chang N -L 1995 Angew. Chem. Int. Ed. Engl. 341555

35. Etter M C, MacDonald J C and Bernstein J 1990 Acta Cryst. B 46256

36. Xue X, Wang X S, Wang L Z, Xiong R G, Abrahams B F, You X Z, Xue Z L and Che C M 2002 Inorg. Chem. 416544 